\title{
Severity scoring system for paediatric FMF
}

Gokhan Kalkan, Erkan Demirkaya and Seza Ozen

In a recent commentary in Nature Reviews Rheumatology, Livneh ${ }^{1}$ discussed paediatric scoring systems for familial Mediterranean fever (FMF; Severity score system for paediatric FMF, Nat. Rev. Rheumatol. 8, 309-310; 2012) in relation to our recent manuscript on the topic. ${ }^{2}$ We consider sharing our opinion with the scientific community as an ethical obligation and, therefore, would like to respond to the criticisms in the Livneh commentary. ${ }^{1}$

One of the key messages of our study was the lack of consistency between the current severity scoring tools for paediatric FMF. However, this finding would not necessarily make studies of severity scoring systems questionable. In this regard, Ozen et al. ${ }^{3}$ examined the environmental effects on the severity of childhood FMF by adjusting Mor ${ }^{4}$ and Pras $^{5}$ scoring systems to children, and did not compare the consistency of different scoring systems, but compared the results of each scoring system according to the country of residence. Ozen et al. ${ }^{3}$ also demonstrated disagreement between the two scoring systems, although they did not specifically mention such finding. For example, the percentage of patients with severe disease in Turkey was $78.2 \%$ based on modified scoring system by Mor and colleagues. However, the percentage of patients with severe disease was only $34.5 \%$ according to modified Pras scoring system.
Another area of criticism in the Livneh commentary was the modification of the colchicine dose, in that it might have biased our results. The clinician's decision regarding the dose of colchicine administered for adults is primarily related to disease severity. However, age and weight are the main determinants of colchicine dosing in children. Therefore, it is evident that the amount of dosing might not be a direct indicator of disease severity for an infant as compared with an adult. Moreover, colchicine dose by itself as a measure of disease severity might not be the best choice in light of current knowledge of pharmacogenetics; increase in colchicine dose used can be due to the pharmacogenetic profile of an individual, rather than an increase in disease severity. In addition, there is a subgroup of patients who do not respond to colchicine but do respond to other therapies (such as as interleukinreceptor blockers). In this regard, severity scoring systems, irrespective of their modification status, will probably miss a patient with severe disease.

Lastly, we are pleased to hear Dr Livneh's support for an international consortium to establish a severity scoring system specifically for children with FMF. The distinct course of the disease in the paediatric age group decreases the utility of the scoring systems of Mor et al. ${ }^{4}$ and Pras et al., ${ }^{5}$ which were originally developed for adults with FMF. There are many qualitative FMF researchers across the Mediterranean basin We will be supportive of any initiative that aims to bring expert experiences together.

Department of Paediatrics, Gazi University, Cocuk Yogun Bakim Unitesi 10.kat Yenimahalle, Ankara 06500, Turkey (G. Kalkan). Division of Paediatric Rheumatology, Gulhane Military Medical Faculty, FMF Arthritis Vasculitis and Orphan disease Research in Paediatric Rheumatology (FAVOR), 06018 Etlik, Ankara, Turkey (E. Demirkaya). Paediatric and Nephrology Unit, Hacettepe University School of Medicine, 06100 Sihhiye, Ankara, Turkey (S. Ozen).

Correspondence to: E. Demirkaya

dottore_erkan@yahoo.com

doi:10.1038/nrrheum.2012.54-c1

\section{Competing interests}

The authors declare no competing interests.

1. Livneh, A. Diagnosis: Severity scoring system for paediatric FMF. Nat. Rev. Rheumatol. 8, 309-10 (2012).

2. Kalkan, G. et al. Evaluation of the current disease severity scores in paediatric FMF: is it necessary to develop a new one? Rheumatology (Oxford) 51, 743-748 (2012).

3. Ozen, S. et al. Disease severity in children and adolescents with familial Mediterranean fever: a comparative study to explore environmental effects on a monogenic disease. Ann. Rheum. Dis. 68, 246-248 (2009).

4. Mor, A. et al. Evaluation of disease severity in familial Mediterranean fever. Semin. Arthritis Rheum. 35, 57-64 (2005).

5. Pras, E. et al. Clinical differences between North African and Iraqi Jews with familial Mediterranean fever. Am. J. Med. Genet. 75 216-219 (1998). 